\title{
Luminosity function of GRBs
}

\author{
S. Sethi ${ }^{1}$ and S. G. Bhargavi ${ }^{2}$ \\ 1 Harish-chandra Research Institute, Jhusi Road, Allahabad 211 019, India \\ e-mail: sethi@mri.ernet.in \\ 2 Indian Institute of Astrophysics, Sarjapur Road, Bangalore 560 034, India \\ e-mail: bhargavi@iiap.ernet.in
}

Received 12 February 2001 / Accepted 29 June 2001

\begin{abstract}
We attempt to constrain the luminosity function of Gamma Ray Bursts (GRBs) from the observed number count-flux relation and the afterglow redshift data. We assume three classes of luminosity functions for our analysis: (a) Log-normal distribution, (b) Schechter distribution, and (c) Scale-free distribution. We assume several models of the evolution of the GRB population for each luminosity function. Our analysis shows that: (a) log-normal is the only luminosity function that is compatible with both the observations. This result is independent of the GRB evolution model, (b) for log-normal function, the average photon luminosity $L_{0}$ and the width of the luminosity function $\sigma$ that are compatible with both the observations fall in the range: $10^{55} \mathrm{~s}^{-1} \lesssim L_{0} \lesssim 10^{56} \mathrm{~s}^{-1}$ and $2 \lesssim \sigma \lesssim 3$, (c) the agreement of observations with other luminosity functions requires the GRB population to evolve more strongly than the evolution of the star-formation rate of the universe.
\end{abstract}

Key words. gamma rays: bursts - cosmology: observations

\section{Introduction}

Recent afterglow observations of GRBs have given important clues about the cosmological nature of GRBs and their environment and luminosities (for details see Kulkarni et al. 2000 and references therein). The afterglow database (see Greiner's home page; Greiner 2000) is sufficiently large that one could now think of using these data for other studies in astronomy. For instance, one could address the issue concerning the luminosity distribution of GRBs using the redshift measurements available in the database. The luminosity distribution of GRBs was hitherto obtainable only from the number count-flux relationship (Piran 1992, 1999; Mao \& Paczyński 1992; Woods \& Loeb 1995; Lubin \& Wijers 1993; Ulmer \& Wijers 1995; Ulmer et al. 1995; Cohen \& Piran 1995; Band et al. 1999; Hakkila et al. 1996; Horack \& Hakkila 1997). The redshift measurement of several GRB afterglows has allowed direct determination of GRB luminosities. The distribution of these luminosities can independently be used to get information about the luminosity function.

In this paper we constrain the luminosity function of GRBs using two approaches: (a) using the redshift data for a sample of 16 GRBs from afterglow measurements; (b) using the number-count $\mathrm{v} / \mathrm{s}$ flux (i.e., $\mathcal{N}-F$ ) relation for GRBs in the current Burst and Transient Source

Send offprint requests to: S. Sethi,

e-mail: sethi@mri.ernet.in
Experiment (BATSE) catalog. In doing so, we consider various luminosity functions (viz. Schechter, scale-free and log-normal) each with "no-evolution" and some of the evolutionary models consistent with the star-formation history of the universe. Our aim is to identify the class of luminosity functions which are consistent with both the $\mathcal{N}-F$ relation for BATSE GRBs and the luminosity distribution of the sample of GRBs whose redshifts are available from the afterglow observations.

In Sect. 2 we briefly review the $\mathcal{N}-F$ method and describe models of luminosity function and GRB number density evolution. In Sect. 3 we discuss the afterglow redshift data and the Likelihood method used for extracting information about the luminosity function parameters from this data. The results are presented and discussed in Sect. 4. Throughout this paper we assume a cosmological model with $\Omega_{\mathrm{m}}=0.3, \Omega_{\Lambda}=0.7, H_{0}=65 \mathrm{~km} \mathrm{~s}^{-1} \mathrm{Mpc}^{-1}$; this model is favoured by recent observations (Perlmutter et al. 1998; de Bernardis et al. 2000; Freedman et al. 2000).

\section{The $\mathcal{N}(>F)-F$ of BATSE GRBs}

For calculating the number of bursts exceeding a given flux $F, \mathcal{N}(>F)$, from BATSE sources we first need to define "flux" of a GRB. We use the BATSE peak photon flux (Paczyǹski 1995) averaged over the trigger time of $1.024 \mathrm{~s}$ as being representative of GRB flux. BATSE reports peak photon flux, $F$, integrated over an energy range 
from $50 \mathrm{kev}$ to $300 \mathrm{kev}^{1}$. This is related to the photon luminosity $L$ (in photons $\mathrm{s}^{-1}$ ) as (see Appendix A for a derivation):

$F=\frac{L(1+z)^{2-\alpha}}{4 \pi D_{\mathrm{L}}^{2}}$

Here $F$ is in units $\mathrm{cm}^{-2} \mathrm{~s}^{-1} . \alpha$ is the spectral index of intrinsic photon luminosity. We take $\alpha=2$ as it provides a reasonable fit to the bursts spectra (for more details and caveats see Band et al. 1993. Note that the spectral index $\alpha$ here is the high-energy spectral index $\beta$ as defined in Band et al. 1993). The luminosity distance $D_{\mathrm{L}}=r(1+z)$, $r$ is the coordinate distance to the GRB at a redshift $z$. For flat cosmologies i.e. $\Omega_{\text {Total }}=1$, it is given by:

$r=H_{0}^{-1} \int_{0}^{z} \frac{\mathrm{d} z^{\prime}}{\left(\Omega_{\mathrm{m}}\left(1+z^{\prime}\right)^{3}+\Omega_{\Lambda}\right)^{1 / 2}}$.

Here the Hubble length $H_{0}^{-1}=9.1 \times 10^{27} h^{-1} \mathrm{~cm} . \Omega_{\mathrm{m}}$ and $\Omega_{\Lambda}$ are the present energy densities (in units of the critical density) of the non-relativistic matter and the cosmological constant, respectively. Observations of SNIa at high redshifts and anisotropies of Cosmic Micro-wave Background Radiation (CMBR) are consistent with a flat cosmological model with $\Omega_{\Lambda} \simeq 0.7$ (Perlmutter et al. 1998; de Bernardis et al. 2000).

In current BATSE catalog peak fluxes are available for 2093 events. We take $F \geq 0.4 \mathrm{~cm}^{-2} \mathrm{~s}^{-1}$ for comparing theoretical predictions with the observed $\mathcal{N}(>F)-F$ from the BATSE catalog (Loredo \& Wasserman 1998). This requirement leaves $\simeq 1790$ burst for our analysis. These GRBs are divided into 170 flux bins for computing the number count-flux relation.

The number count $\mathcal{N}(>F)$ can be expressed as:

$\mathcal{N}(>F)=4 \pi \int_{0}^{z_{\max }} \mathrm{d} r r^{2} \int_{4 \pi D_{\mathrm{L}}^{2} F}^{\infty} \mathrm{d} L n(L, z)(1+z)^{-\alpha}$

Here

$n(L, z)=\phi_{*}(z) \phi(L) \mathrm{d} L$

is the comoving number density of GRBs in a given luminosity range $L$ to $L+\mathrm{d} L$ at a given redshift. $\phi(L)$ is the luminosity function, defined such that $\int_{0}^{\infty} \phi(L) \mathrm{d} L=1$. $\phi_{*}(z)=\phi_{*}(0) \times(1+z)^{\gamma}$ is the total comoving number density of GRBs at redshift $z$. The factor of $(1+z)^{-\alpha}$ in Eq. (3) gives the "k-correction" which corrects for the difference between the emitted and the observed wavelength.

The functional form of the luminosity function of the GRBs is unknown. In the framework of fireball models, the luminosity function of GRBs is expected to be broad with luminosity width of nearly 2 orders of magnitude (Kumar \& Piran 1999). For our study, we assume several different

\footnotetext{
1 For more details see
}

http://gammaray.msfc.nasa.gov/batse/data/ luminosity functions, $\phi(L)$ :

Log-normal distribution function:

$$
\phi(L)=\frac{\exp \left(-\sigma^{2} / 2\right)}{\sqrt{2 \pi \sigma^{2}}} \exp \left[-\left(\ln \left(L / L_{0}\right)\right)^{2} /\left(2 \sigma^{2}\right)\right] \frac{1}{L_{0}}
$$

$\sigma$ and $L_{0}$ are the width and the average luminosity of the luminosity function, respectively (for previous use of this luminosity function for GRB studies see e.g. Woods \& Loeb 1995). The log-normal distribution is also representative of the luminosity function of spiral galaxies (for details see Bingelli et al. 1988).

\section{Schechter distribution:}

$\phi(L)=A\left(\frac{L}{L_{*}}\right)^{-\beta} \exp \left(-L / L_{*}\right) \frac{1}{L_{*}}$

$A$ is a normalizing constant. The entire galaxy population can be roughly represented by this function (for details see Bingelli et al. 1988). We restrict ourselves to $\beta \leq 1$ in this paper.

Scale-free luminosity function:

$\phi(L)=A\left(\frac{L}{L_{*}}\right)^{-\beta} \frac{1}{L_{*}}$ for $L_{\min } \leq L \leq L_{\max }$

$A$ is a normalizing constant. Several authors have used this class of luminosity functions for GRB analyses (see e.g. Schaefer 2000).

$\mathcal{N}(>F)$ depends on a number of parameters: (a) cosmological parameters through $D_{\mathrm{L}}$ and $r$. We fix the cosmological parameters to their most favoured values. (b) $z_{\max }$, the maximum redshift of GRBs, (c) $\phi_{*}(z)$, which gives the redshift dependence of the GRB population, (d) the parameters of the luminosity function. Our aim is to obtain the most-favoured values of the luminosity function parameters. After fixing the cosmological model and the GRB spectral index, the most important remaining uncertainty comes from the evolution of the GRB population. One obvious choice is the "no evolution" model. In this model, $\phi_{*}(z)$ is independent of $z$. However afterglow observations give circumstantial evidence that GRBs might be associated with star-forming regions (Kulkarni et al. 2000 and references therein). Such an association might mean that GRB population trace the star-formation history of the universe. Therefore we also consider several other models, which are consistent with the star-formation history of the universe:

Model I: No evolution model

Model II: The GRB population evolves as the luminosity density at $1600 \AA$, which is a good tracer of starformation history (Madau et al. 1998; Madau et al. 1996). In this case the comoving number density of GRBs increases by a factor of $\simeq 10$ from $z \simeq 0$ to $z \simeq 1.5$ (Lilly et al. 1996) and then decreases approximately $\propto(1+z)^{-1}$ at higher redshifts (Fig. 3 of Madau et al. 1998). 
Model III: To account for the possible dust-extinction in the star-forming regions for $z \geq 1.5$ (Conolly et al. 1997), we assume that the star-formation rate grows for $z \leq 1.5$ as in the previous model, but remains constant at the value of $z=1.5$ till $z \simeq 3$, and then declines as $\propto(1+z)^{-1}$ at larger redshifts.

Model IV: We consider an extreme model that, given the uncertainty in the evolution of the star-formation rate at $z \geq 1.5$, is barely consistent with the star-formation history of the universe. In this model, the comoving number density of GRB population evolves $\propto(1+z)^{3.5}$ for $z \lesssim 1.5$ (Lilly et al. 1996). The comoving number density remains at the value of $z=1.5$ for $z \leq 5$ and then it gradually declines at $\propto(1+z)^{-0.5}$. In this model much of star-formation at redshifts $\gtrsim 1.5$ occurs in regions highly shrouded by dust which allows a high star-formation rate to be compatible with the star-formation rate observed in the Hubble Deep Field UV drop-out galaxies. However, further increase in the star-formation rate, which must be accompanied by a suitable increase in the dust content, might be incompatible with the observation of Far Infra-red background (Puget et al. 1996; Guiderdoni et al. 1997).

\section{Observed GRB redshifts}

In Table 1 we list the redshifts of GRBs used to calculate the luminosity distribution of GRBs. The GRB at $z=0.008$ (GRB 980425) has been excluded from this list, as it is probably associated with a supernova (Galama et al. 1998) and therefore corresponds to a different population of GRBs (Kulkarni et al. 2000). Three GRBs whose redshifts are not certain (GRB 980326: $z \simeq 1$; GRB 980329: $z \leq$ 3.5; GRB 990507: $z \simeq 0.25$ ) are also excluded.

Gamma-ray fluxes (photons $\mathrm{cm}^{-2} \mathrm{~s}^{-1}$ ) corresponding to these 16 GRBs are listed in Table 1: eight of these are taken from BATSE flux table (integrated over 1024 milliseconds). The gamma-ray fluxes of remaining GRBs, triggered either by BeppoSAX or IPN satellites are in different energy band and therefore had to be extrapolated to energy range of BATSE. The band-pass conversion is achieved using photon luminosity spectral index, $\alpha=2$ (see Appendix B). We adopt the values from Schaefer (2000) for three of the GRBs.

From the measured redshifts and observed $\gamma$-ray fluxes, the total energies of GRBs, assuming isotropic emission, is in the range $10^{51-54} \mathrm{erg}$. The production of such large energies pose serious problems in theoretical modelling. One of the proposals to resolve the energy crisis was to have the emission collimated into jets, which would lower the total energies by a factor of few hundreds (Rhoads 1999; Piran 1999). The GRB afterglow observations have shown evidence for beaming in the light curves in 5 cases: GRB 990123 (Holland 2000; Kulkarni 1999), GRB 990510 (Holland 2000; Harrison 1999), GRB 991216 (Halpern 2000),

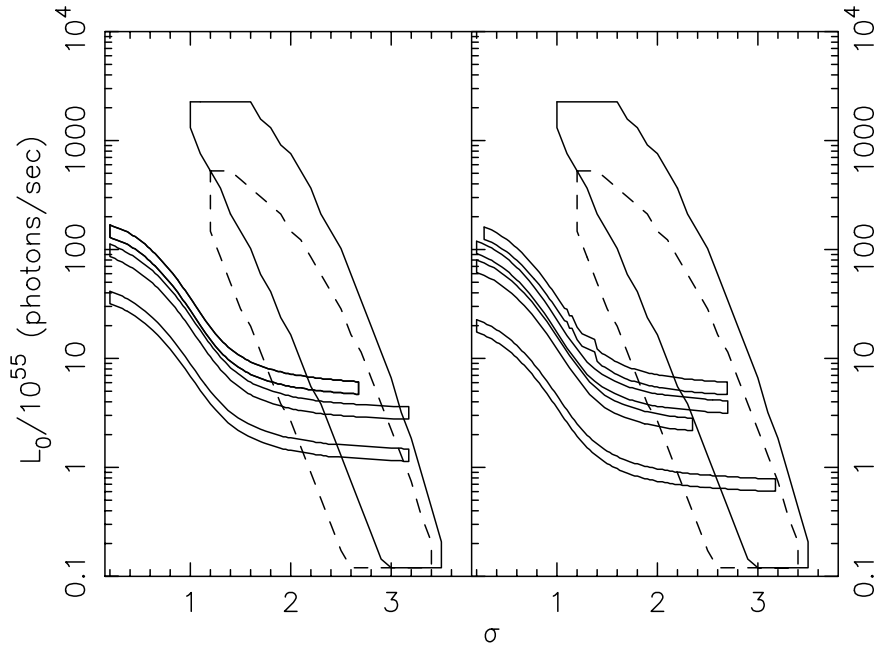

Fig. 1. The results for the Log-normal luminosity function are shown. Right panel: results are shown for the full GRB sample. The bigger regions in the center enclose the allowed region from the afterglow observations. The region with dotted lines corresponds to a run where beaming corrections are applied. The 4 contours on the left side represent the region of K-S probability $P_{\mathrm{ks}}>0.01$ for the consistency between observed and theoretical number count-flux relation. They correspond, from bottom to top (with increasing luminosity) to four models 1-4 respectively of GRB evolution described in the text. Left panel: results are shown for the GRB sample with GRB duration (in T90) exceeding $2 \mathrm{~s}$. Model 4 of GRB evolution is not shown.

GRB 000301c (Sagar et al. 2000; Berger et al. 2000) and GRB 000926 (Price et al. 2000). The opening angle $\theta_{\circ}$ of the jet may be calculated from Eq. (1) of Sari et al. (1999) knowing the break in light curve. The beaming factor in Table 1 . is $\approx 2 / \theta_{\circ}^{2}$ and has been used to apply the corrections in luminosities in 5 cases.

The probability that burst of a given flux will occur in a redshift range $z$ to $z+\mathrm{d} z$ is given by:

$p(z) \mathrm{d} z=\phi(L) \times \frac{\mathrm{d} L}{\mathrm{~d} z} \mathrm{~d} z$

$\phi(L)$ is given by Eqs. (5)-(7), and $L$ for a given flux $F$ and redshift is determined by Eq. (1).

The likelihood that the observed GRB luminosities were drawn from a given luminosity function is:

$\mathcal{L}\left(a_{k}\right)=\prod_{i=1}^{16} p\left(z_{i}, F_{i}\right)$.

We maximize the likelihood function with respect to the parameters of the luminosity function, $a_{k}$, e.g. $a_{k}=$ $\left\{L_{0}, \sigma\right\}$ for the Log-normal luminosity function. It should be noted that parameters estimated using this method do not depend on the number density $\phi_{*}(z)$ of the GRBs.

\section{Results}

We show our results for the log-normal luminosity function in Fig. 1 . We plot the allowed region in the $L_{0}-\sigma$ plane 
Table 1. Redshifts and luminosities of Afterglow GRBs.

\begin{tabular}{lllllll}
\hline GRB & $\begin{array}{l}\text { BATSE } \\
\operatorname{tr} \#\end{array}$ & $\begin{array}{l}\text { Redshift } \\
z\end{array}$ & $\begin{array}{l}\text { Photon flux } \\
\mathrm{ph} / \mathrm{s} / \mathrm{cm}^{2}\end{array}$ & $\begin{array}{l}\text { Luminosity } \\
\mathrm{ph} / \mathrm{s}\end{array}$ & $\begin{array}{l}\text { Beaming } \\
\text { factor* }^{*}\end{array}$ & $\begin{array}{l}\text { Ref. for } \\
\text { photon flux }\end{array}$ \\
\hline GRB 970228 & - & 0.695 & 10.0 & $2.34 \times 10^{58}$ & - & 1 \\
GRB 970508 & 6225 & 0.835 & 0.969 & $3.56 \times 10^{57}$ & - & 2 \\
GRB 970828 & 6350 & 0.958 & 1.5 & $7.74 \times 10^{57}$ & - & 1 \\
GRB 971214 & 6533 & 3.418 & 1.955 & $2.27 \times 10^{59}$ & - & 2 \\
GRB 980613 & - & 1.096 & 0.5 & $3.60 \times 10^{57}$ & - & 1 \\
GRB 980703 & 6891 & 0.966 & 2.398 & $1.26 \times 10^{58}$ & - & 2 \\
GRB 990123 & 7343 & 1.6 & 16.41 & $3.0 \times 10^{59}$ & 300 & 2 \\
GRB 990506 & 7549 & 1.31 & 18.56 & $2.08 \times 10^{59}$ & - & 2 \\
GRB 990510 & 7560 & 1.619 & 8.16 & $1.54 \times 10^{59}$ & 300 & 2 \\
GRB 990712 & 7647 & 0.434 & 11.64 & $8.56 \times 10^{57}$ & - & 2 \\
GRB 991208 & - & 0.706 & 11.2 & $2.72 \times 10^{58}$ & - & 3 \\
GRB 991216 & 7906 & 1.02 & 67.5 & $4.06 \times 10^{59}$ & 200 & 2 \\
GRB 000131 & - & 4.5 & 1.5 & $3.35 \times 10^{59}$ & - & 3 \\
GRB 000301c & - & 2.03 & 1.32 & $4.34 \times 10^{58}$ & 90 & 3 \\
GRB 000418 & - & 1.118 & 3.3 & $2.5 \times 10^{58}$ & - & 3 \\
GRB 000926 & - & 2.066 & 10.0 & $3.45 \times 10^{59}$ & 120 & 3 \\
\hline
\end{tabular}

* References for beaming factor follow: GRB 990123: Holland et al. (2000); GRB 990510: Holland et al. (2000), Harrison et al. (1999); GRB 991216: Halpern et al. (2000); GRB 000301c: calculated for $t_{\mathrm{b}}=4.1$ days from Bhargavi \& Cowsik (2000) using Eq. (1). of Sari et al. (1999); GRB 000926: Price et al. (2000).

** The references for fluxes are as follows: 1. Schaefer (2000); 2. BATSE catalog; 3. conversion made by applying band-pass corrections as explained in Appendix B.

from both the observed $\mathcal{N}(>F)-F$ from BATSE catalog and the afterglow redshift data. The allowed region, by requiring consistency between the observed $\mathcal{N}(>F)-F$ relation and the theoretical number counts (Eq. (3)), corresponds to the area in which the K-S probability $P_{\mathrm{ks}} \geq 0.01$ (see e.g. Press et al. 1992 for details on the K-S test).

The best fit value of $\sigma$ and $L_{0}$ from the afterglow redshift data are: $\sigma=1.7$ and $L_{0}=3 \times 10^{57} \mathrm{~s}^{-1}$ without the beaming corrections and $\sigma=2$ and $L_{0}=2 \times 10^{56} \mathrm{~s}^{-1}$ with the beaming corrections. We show in Fig. 1 the region within which the value of the likelihood function is $\geq 10^{-4}$ times the value at the maximum. This corresponds roughly to the $99 \%$ confidence level for a two-parameter fit (Press et al. 1992). (We do not calculate the joint confidence levels using the usual Fisher matrix approach because the Likelihood function is not a joint Gaussian distribution and also because the Likelihood function is very broad near the maximum).

Results for other assumed luminosity functions are shown in Figs. 2-4, using the same criteria for showing the allowed regions as given in the preceding paragraph. The results are shown for only two representative scale-free models. The best-fit values of parameters from GRB redshift data are: $\beta=0.6, L_{*}=3 \times 10^{59} \mathrm{~s}^{-1}$ without beaming correction and $\beta=0.6, L_{*}=2 \times 10^{59} \mathrm{~s}^{-1}$ with beaming correction (Schechter luminosity function); $\beta=0.65, L_{*}=5 \times 10^{56} \mathrm{~s}^{-1}$ without beaming corrections and $\beta=1.05, L_{*}=3.8 \times 10^{56} \mathrm{~s}^{-1}$ (scale-free model with $L_{\min } \simeq L_{*}$ and $\left.L_{\max } \simeq 10^{3} L_{*}\right) ; \beta=0.65, L_{*}=$ $6 \times 10^{57} \mathrm{~s}^{-1}$ without beaming correction and $\beta=1.15$,

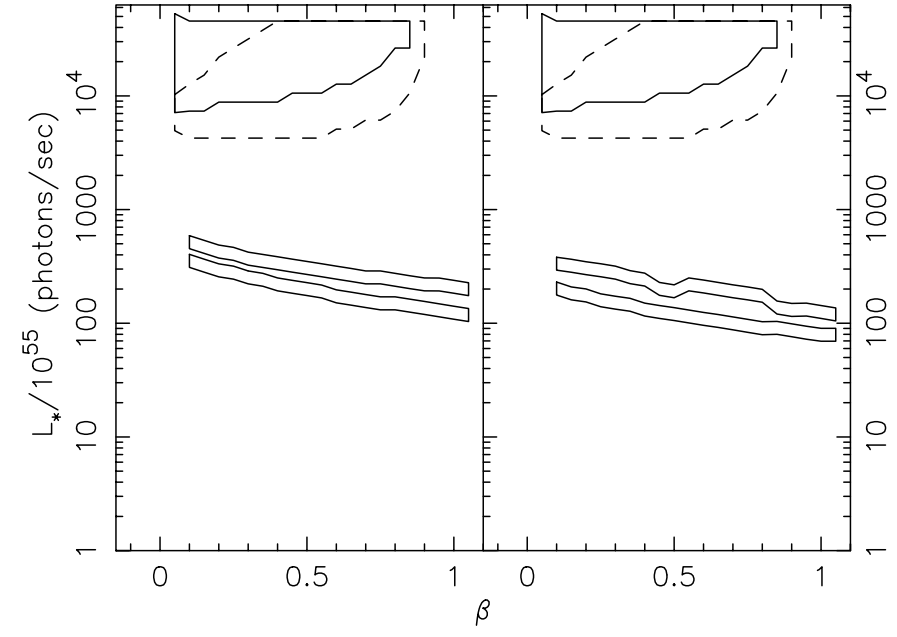

Fig. 2. The results for the Schechter luminosity function are shown. The right and left panels correspond to the full sample and the long duration sample (T90 $\geq 2 \mathrm{~s}$ ), respectively. The bigger regions at the top of the figure come from the afterglow observations. The region inside the solid (dashed) curves correspond to no (with) beaming correction. The smaller regions at the center are from the K-S test for the number count-flux relation. These correspond, with increasing photon luminosity, to GRBs evolution models III and IV discussed in the text. The allowed regions for models I and II fall below the allowed regions for the models shown.

$L_{*}=1.2 \times 10^{58} \mathrm{~s}^{-1}$ with beaming correction (scale-free model with $L_{\min } \simeq 3 \times 10^{-2} L_{*}$ and $L_{\max } \simeq 10^{2} L_{*}$ ).

As seen in the figures, it is possible to explain the observed $\mathcal{N}(>F)-\mathrm{F}$ relation using any of the luminosity 


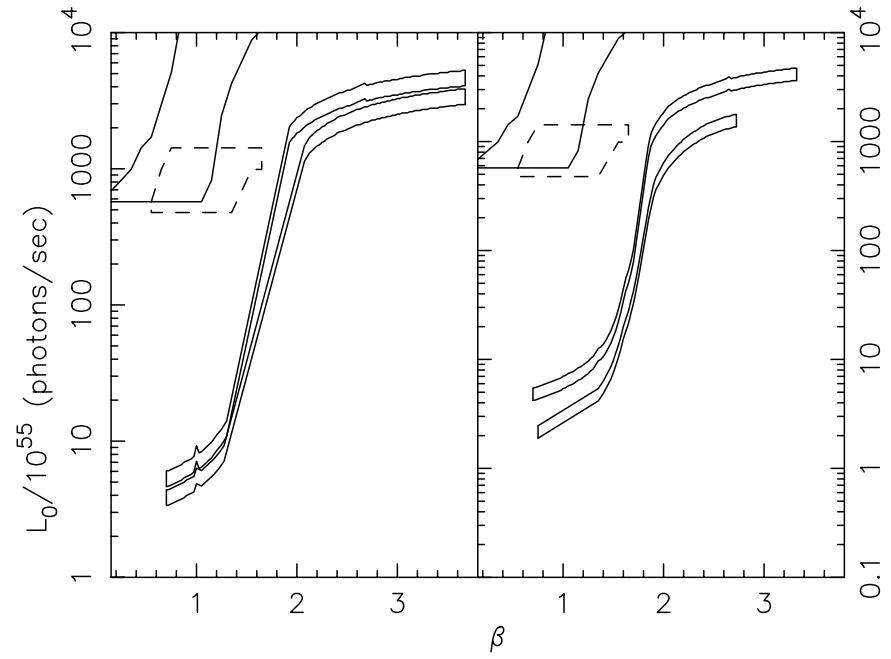

Fig. 3. The results for the scale-free luminosity function are shown. In this model $L_{\min }=3 \times 10^{-2} L_{*}$ and $L_{\max }=100 L_{*}$. The right and left panels correspond to the full sample and the long duration sample (T90 $\geq 2 \mathrm{~s})$, respectively. The regions at the left of the figure come from afterglow observations; the region inside the solid (dashed) curves correspond to no (with) beaming correction. The thin regions are from the K-S test for the number count-flux relation. These correspond, with increasing photon luminosity, to GRBs evolution models III and IV discussed in the text. The allowed regions for models I and II fall below the allowed regions for the models shown.

functions we assume. Further the allowed regions do not strongly constrain either the luminosity of the GRB or the width of the luminosity function (Loredo \& Wasserman 1998; Schmidt 2000). For any luminosity function the allowed range of luminosities span atleast a decade depending on the evolution of GRB population (Fig. 1). It can be noticed that stronger evolution in the GRB population results in a higher average luminosity (Schaefer 2000). $z_{\max }$, the maximum redshift of GRBs is not an important parameter as long as $z_{\max } \gtrsim 5$. It is because even the faintest bursts we consider in our analysis $\left(F \simeq 0.4 \mathrm{~cm}^{-2} \mathrm{~s}^{-1}\right)$ come from $z \lesssim 5$ for much of the allowed luminosity range.

The GRB redshift data give a large range of photon luminosities $\left(10^{57} \mathrm{~s}^{-1} \lesssim L \lesssim 10^{59} \mathrm{~s}^{-1}\right.$ in photons $\left.\mathrm{s}^{-1}\right)$. Therefore it is natural to expect that the underlying luminosity function is broad. This is clearly seen in the figures. It should be noted that the GRB luminosities implied by the $\mathcal{N}(>F)-$ F relation are not at variance with the observed GRB luminosities. However, as seen in the figures, these two observations imply two different set of luminosity function parameters in most cases.

The log-normal is the only luminosity function that is compatible with both BATSE number count and the afterglow redshift data. This agreement is independent of the GRB evolution model. The agreement between the two data sets requires $10^{55} \mathrm{~s}^{-1} \lesssim L_{0} \lesssim 10^{56} \mathrm{~s}^{-1}$ and $2 \lesssim \sigma \lesssim 3$. For other luminosity functions the agreement between the two observations becomes better as GRB evolution becomes stronger (Figs. 2-4). However

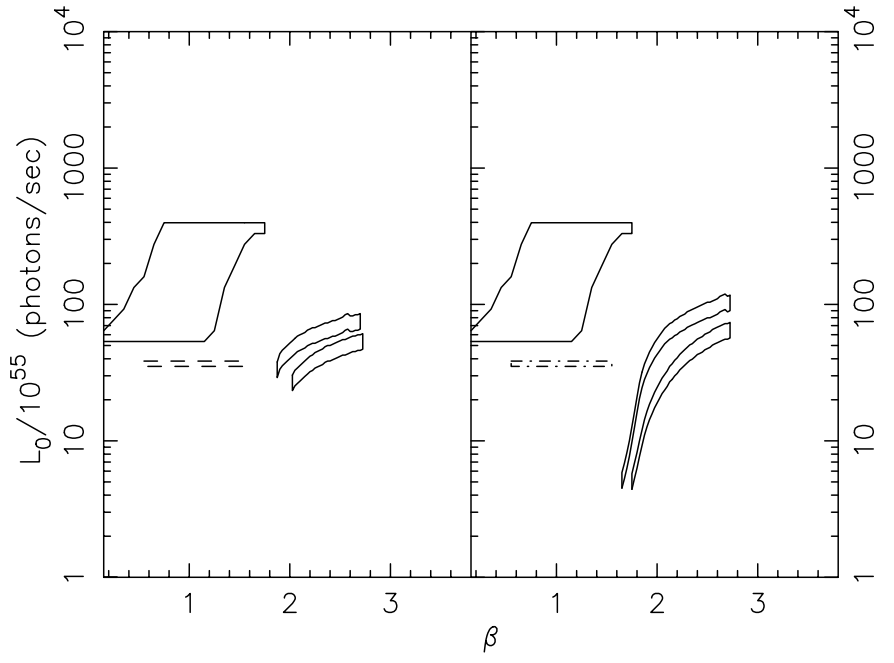

Fig. 4. The results for the scale-free luminosity function are shown. In this model $L_{\min }=L_{*}$ and $L_{\max }=1000 L_{*}$. The right and left Panels correspond to the full sample and the long duration sample (T90 $\geq 2 \mathrm{~s}$ ), respectively. The regions at the left of the figure come from afterglow observations with no (with) beaming correction corresponding to solid (dot-dashed) curves. Thin regions in the center of the figure are from the K-S test for the number count-flux relation. These correspond with increasing photon luminosity, to GRBs evolution model III and IV discussed in the text. The allowed regions for models I and II fall below the allowed regions for the models shown.

the requirement that the two regions overlap can be fulfilled only if the GRBs evolve at a rate much stronger than the star-formation rate of the universe.

It is seen in Figs. 1-4 that the beaming correction does not make any quantitative difference to our results. In almost all the cases the $99 \%$ region from the afterglow data becomes smaller after the beaming correction, but it is difficult to draw any conclusions from it. A particularly interesting case is one of the scale-free models (Fig. 4) in which the beaming correction reduces the area of the $99 \%$ region quite dramatically. However this is owing to the fact that the beaming correction increases the luminosity width of the observed GRB afterglows, and the model in question has a luminosity width of 1000 which matches the luminosity width of the GRBs. Therefore only a very small range of $L_{*}$ is compatible with observations.

Selection effects: One possible reason for the discrepancy/agreement between the number count-flux and GRB redshift data results could be selection effects. Most of the redshift determinations have been possible owing to the detection of X-ray counterpart of the GRB by BeppoSAX. This results in two types of selection effects: (a) BeppoSAX is not sensitive to burst duration $\leq 1 \mathrm{~s}$ (Feroci et al. 1999), (b) the GRBs detected by BeppoSAX might be X-ray selected though Feroci et al. (1999) emphasize it is unlikely.

The burst-duration selection effect might mean the GRBs for which the redshifts have been determined belong to a different population of GRBs. The burst duration 
distribution for the BATSE bursts is observed to be bimodal (Fishman \& Meegan 1995), which strongly suggests that long- and short-duration bursts are different populations of GRBs. In light of this it is usual to expect that these two might have different luminosity functions. To check for this effect we make a sub-catalog of GRB bursts with duration $\geq 2 \mathrm{~s}$ (measured in T90). As seen in Figs. 1 to 4 , the range of allowed luminosity function parameters from the $\mathcal{N}(>F)-F$ relation of this sub-catalog are qualitatively similar to those for the entire catalog, and our results are not sensitive to this selection criterion.

Another selection effect is the Malmquist bias (see e.g. Sandage 1993 for a discussion and relevant references): if the luminosity function of sources is calculated from a magnitude-limited sample then the average luminosity of the estimated luminosity function exceeds the true mean of the underlying population. Other moments of the luminosity function (variance, etc.) are also affected. For GRBs, unlike other astronomical sources, it is difficult to establish the criterion of source selection i.e. it is not completely clear that the source whose luminosities have been established are selected randomly from a flux-limited sample. For the purposes of this paper we have taken this assumption to be true. To assess the effect of Malmquist bias, we calculate, for the luminosity functions considered in this paper, the average luminosity $\langle L\rangle_{\mathrm{f}}$ and its variance for a flux-limited sample (see e.g. Sandage 1993). The flux limit of the sample is taken to be the minimum flux for which the luminosity has been determined $\left(\simeq 0.5 \mathrm{~cm}^{-2} \mathrm{~s}^{-1}\right.$; Table 1$)$. In addition to flux, $\langle L\rangle_{\mathrm{f}}$ has dependence on the parameters of the luminosity function and evolutionary properties of the whole population. We find that, within the range of relevant luminosity function parameters and the evolution of GRB population:

1. The average luminosity of the flux-limited sample exceed the true mean by a factor of two to three;

2. The variance of the flux-limited sample is within $40-50 \%$ of the true variance.

This is well within the range of $99 \%$ confidence contours from the afterglow observations (Figs. 1 to 4 ). It should be pointed out that to get complete information about the luminosity function, we need to calculate all the higher moments. However, we note that uncertainties in the first few moments are far below the range of luminosity function parameters allowed by the likelihood analysis of the afterglow data (Figs. 1 to 4). This suggests that errors in the luminosity function parameter are more due to the smallness of the sample than the Malmquist bias, and our conclusion are not affected by this bias.

To sum up: of the four luminosity functions considered here, log-normal is the only luminosity function that is consistent with both the BATSE number count-flux relation and afterglow redshift data, independent of the evolution of the GRB population. Notwithstanding the selection effects, the other luminosity function can be consistent with both observations only if the GRBs evolution far exceeds the evolution of the star-formation rate in the universe.

\section{Appendix A}

Here we give a brief derivation of Eq. (1). Assume that a source at a redshift $z$ is emitting with photon luminosity $L_{\nu}$ (photons $\mathrm{s}^{-1} \mathrm{~Hz}^{-1}$ ), with intrinsic spectrum:

$$
L_{\nu}=L_{\mathrm{f}}\left(\frac{\nu}{\nu_{\mathrm{f}}}\right)^{-\alpha}
$$

Here $\nu_{\mathrm{f}}$ is some fiducial frequency. The received photon flux (photons $\mathrm{cm}^{-2} \mathrm{~s}^{-1} \mathrm{~Hz}^{-1}$ ) is:

$F_{\nu_{0}}=\frac{L_{\nu}}{4 \pi r^{2}}$.

Here $r$, defined in Eq. (2), is the coordinate distance to the source from the observer at the present epoch and $\nu_{0}$ is the observed frequency. Note that there are no factors of $(1+z)$ in the denominator of Eq. (11). Integrating the flux over the observed band-pass $(50 \mathrm{kev}$ to $300 \mathrm{kev}$ for BATSE), and using $\nu / \nu_{0}=(1+z)$, we get:

$F \equiv \int F_{\nu_{0}} \mathrm{~d} \nu_{0}=\frac{L_{\mathrm{f}}(1+z)^{-\alpha}}{4 \pi r^{2}} \int\left(\frac{\nu_{0}}{\nu_{\mathrm{f}}}\right)^{-\alpha} \mathrm{d} \nu_{0}$

Defining

$L=L_{\mathrm{f}} \int\left(\frac{\nu_{0}}{\nu_{\mathrm{f}}}\right)^{-\alpha} \mathrm{d} \nu_{0}$,

and using $D_{\mathrm{L}}=(1+z) r$, we obtain Eq. (1).

\section{Appendix B}

Throughout this paper we take the "flux" to mean the photon flux averaged over 1.024 s BATSE trigger, in the energy range 50-300 kev. Out of 16 afterglows whose redshifts have been determined, only 8 have known BATSE fluxes. In the other cases, the gamma-ray fluxes are observed in other frequency bands by either BeppoSAX or IPN satellites. To be consistent with our definition of flux, we extrapolate the observed fluxes from the observed band to the BATSE band. We briefly describe the method of this extrapolation in this Appendix. It should be pointed out that the difference of trigger time between BATSE and other instruments must also be taken into account to extrapolane fluxes. However, we apply only band-pass corrections.

Assume that a source at redshift $z$ is emitting with a luminosity $L_{\nu}\left(\mathrm{erg} \mathrm{s}^{-1} \mathrm{~cm}^{-2}\right)$ and spectrum:

$L_{\nu}=L_{\mathrm{f}}\left(\frac{\nu}{\nu_{\mathrm{f}}}\right)^{-\beta}$

$\nu_{\mathrm{f}}$ is some fiducial frequency. The observed flux at the frequency $\nu_{0}=\nu /(1+z)$ is:

$F_{\nu_{0}}=\frac{L_{\nu}}{4 \pi r^{2}(1+z)}$ 
The flux integrated over a band-pass between frequencies $\nu_{1}$ and $\nu_{2}$ is given by:

$F\left(\nu_{1}, \nu_{2}\right) \equiv \int_{\nu_{1}}^{\nu_{2}} F_{\nu_{0}} \mathrm{~d} \nu_{0}=\frac{F(z)}{\nu_{\mathrm{f}}^{-\beta}} \int_{\nu_{1}}^{\nu_{2}}\left(\frac{\nu_{0}}{\nu_{\mathrm{f}}}\right)^{-\beta} \mathrm{d} \nu_{0}$,

with the definition $F(z)=L_{\mathrm{f}}(1+z)^{-\beta-1} /\left(4 \pi r^{2}\right)$. We assume $\beta=1$ in this paper (the spectral index $\alpha$ for the photon luminosity is related to $\beta$ as $\alpha=\beta+1$ ). This gives:

$F\left(\nu_{1}, \nu_{2}\right)=\frac{F(z)}{\nu_{\mathrm{f}}^{-1}} \log \left(\nu_{2} / \nu_{1}\right)$

Equation (17) can be used to convert flux observed in any band-pass to the BATSE band-pass.

The photon luminosity $\left(L_{\nu}^{\mathrm{p}}\right)$ is related to the energy luminosity as: $L_{\nu}^{\mathrm{p}}=L_{\nu} /(h \nu), h$ being the Planck's constant. This relation and the methods described above as well as in the previous appendix can be used to get the conversion between the band-pass integrated photon flux $(F)$ and the energy flux $\left(F\left(\nu_{1}, \nu_{2}\right)\right)$. The relation is (for $\beta=1)$ :

$F=F\left(\nu_{1}, \nu_{2}\right) \frac{\nu_{1}^{-1}-\nu_{2}^{-2}}{h \log \left(\nu_{2} / \nu_{1}\right)}$.

Acknowledgements. We thank J. Greiner, R. Cowsik, and D. Hartmann for reviewing the manuscript and giving useful comments; A. Loeb for suggesting us to make beaming corrections; D. Bhattacharya for useful discussions and M. R. Kippen for information on our queries about BATSE fluxes. We also thank the referee for helpful comments.

\section{References}

Band, D., et al. 1993, ApJ, 413, 281

Band, D., Hartman, D., \& Schaefer, B. 1999, ApJ, 514, 862

Berger, E., et al. 2000 [astro-ph/0005465]

Bhargavi, S. G., \& Cowsik, R. 2000, ApJ, 545, L77

Bingelli, B., Sandage, A., \& Tamman, G. A. 1988, ARA\&A, 26, 509
Connolly, A. J., Szalay, A. S., Dickinson, M., SubbaRao, M. U., \& Brunner, R. J. 1997, ApJ, 486, L11

Cohen, E., \& Piran, T. 1995, ApJ, 444, L25

de Bernardis, P., et al. 2000, Nature, 404, 995

Feroci, M., et al. 1999, A\&AS, 138, 407

Fishman, G. J., \& Meegan, C. A. 1995, ARA\&A, 33, 415

Freedman, W., et al. 2000 [astro-ph/0012376]

Galama, T. J., et al. 1998, Nature, 395, 670

Greiner, J. 2000, http://www .aip.de/People/JGreiner

Guiderdoni, B., et al. 1997, Nature, 390, 257

Hakkila, J., et al. 1996, ApJ, 462, 125

Halpern, J. P., et al. 2000 [astro-ph/0006206]

Harrison, F. A., et al. 1999, ApJ, 523, L121

Holland, S., et al. 2000 [astro-ph/0010196]

Horack, J. M., \& Hakkila, J. 1997, ApJ, 479, 371

Kulkarni, S. R., et al. 2000 [astro-ph/0002168]

Kulkarni, S. R., et al. 1999, Nature, 398, 389

Kumar, P., \& Piran, T. 1999 [astro-ph/9909014]

Lilly, S., Le Fèvre, O., Hammer, F., Crampton, D. 1996, ApJ, 460, L1

Loredo, T. J., \& Wasserman, I. 1998, ApJ, 502, 75

Lubin, L. M., \& Wijers, R. A. M. J. 1993, ApJ, 418, L9

Madau, P., Pozzetti, L., \& Dickinson, M. 1998, ApJ, 498, 106

Madau, P., Ferguson, H. C., Dickinson, M., et al. 1996, MNRAS, 283, 1388

Mao, S., \& Paczyński, B. 1992, ApJ, 388, L45

Paczyǹski, B. 1995, PASP, 107, 1167

Perlmutter, S., et al. 1999, ApJ, 517, 565

Piran, T. 1992, ApJ, 389, L45

Piran, T. 1999, Phys. Rep., 314, 575

Press, W. H., Teukolsky, S., Vellering, W. T., \& Flannery, B. P. 1992, Numerical recipes in fortran (Cambridge University Press)

Price, P. A., et al. 2000 [astro-ph/0012303]

Puget, J.-L., et al. 1996, A\&A, 308, L5

Rhoads, J. E. 1999, ApJ, 525, 737

Sagar, R., et al. 2000, BASI, 28, 499

Sandage, A. 1993, in Saas-Fee advance course 23, ed. B. Bingelli, \& R. Buser

Sari, R., et al. 1999, ApJ, 519, L17

Schmidt, M. 2000 [astro-ph/0001121]

Schaefer, B. 2000, ApJ, 532, L21

Ulmer, A., \& Wijers, R. A. M. J. 1995, ApJ, 439, 303

Ulmer, A., Wijers, R. A. M. J., \& Fenimore, E. E. 1995, ApJ, 440, L9

Woods, E., \& Loeb, A. 1995, ApJ, 453, 583 